\title{
Factores que influyen sobre el valor económico de la industria de confecciones en Colombia ${ }^{1}$
}

\author{
Factors that influence economic value in the Colombian apparel industry
}

\author{
Jorge Alberto Rivera-Godoy \\ Doctor en Ciencias Económicas y Empresariales, profesor titular, Universidad del Valle, \\ Cali - Colombia, jorge.rivera@correounivalle.edu.co \\ Lizeth Carolina Mamián-Cerón \\ Administradora de empresas y Contadora Pública, Universidad del Valle, Cali - Colombia, \\ lizeth.mamian.ceron@correounivalle.edu.co \\ Cristhian Andrés Rojas-Zapata \\ Contador Público, Universidad del Valle, Cali - Colombia, \\ cristhian.rojas@correounivalle.edu.co
}

\begin{abstract}
Cómo citar / How to cite
Rivera-Godoy, J., Mamián-Cerón, L., \& Rojas-Zapata, C. (2019). Factores que influyen sobre el valor económico de la industria de confecciones en Colombia. Revista CEA, 5(9), 131-146. https://doi.org/10.22430/24223182.1311
\end{abstract}

Recibido: 8 de noviembre de 2018

Aceptado: 27 de diciembre de 2018

\section{Resumen}

La investigación que se sintetiza en este artículo tiene como objetivo conocer los factores que influyen sobre el valor económico de la industria de confecciones en Colombia, durante el período 2010-2016, siguiendo como metodología un análisis de los indicadores contables y de valor económico agregado que miden su crecimiento, eficiencia, eficacia y efectividad.

Los indicadores contables evidencian que esta industria fue exitosa porque creció y generó rentabilidades contables en cada uno de los años; la variabilidad anual de su efectividad dependió principalmente de la eficacia en la gestión de costos y gastos. Sin embargo, se encontró que destruyó valor económico agregado (EVA) en cada año porque el costo de los recursos financieros fue superior a la rentabilidad del activo neto operacional, lo cual arrojó como saldo un valor de mercado agregado negativo en el septenio. La destrucción de EVA fue fluctuante, resultado de variaciones en la utilidad operacional después de impuestos y en el costo de capital presentadas bajo un continuo crecimiento

\footnotetext{
${ }^{1}$ Artículo resultado de la línea de investigación de «Evaluación del desempeño financiero de empresas del sector real en Colombia», que adelanta el Grupo de Investigación en Generación de Valor Económico (GIGVE), de la Universidad del Valle (categoría C de Colciencias).
} 
del activo neto operacional. Los hallazgos podrían fortalecerse si se realizaran estudios similares para grupos de empresas más homogéneos en cuanto al tamaño, edad y ubicación geográfica.

Palabras clave: desempeño financiero, indicadores financieros de eficiencia, industria de confecciones en Colombia, Valor Económico Agregado (EVA), Valor de Mercado Agregado.

\begin{abstract}
This work aims at identifying the factors that influenced economic value in the Colombian apparel industry from 2010 to 2016. Financial and economic value-added indicators are analyzed to measure the growth, efficiency, efficacy, and effectiveness of such sector.

Financial indicators reveal that the industry was successful because it grew and produced economic yields every year; the annual variability of its effectiveness mainly depended on the efficacy of cost and expense management. However, some Economic Value Added (EVA) was destroyed because the cost of the financial resources exceeded the profitability of the net operating assets, which produced a negative market value added during the seven-year period. EVA destruction was fluctuating, the result of variations in operating profit after tax and capital cost in a context of continuously growing net operating assets. These findings could be confirmed if similar studies were conducted with more homogeneous groups of companies in terms of size, age, and geographic location.
\end{abstract}

Keywords: financial performance, financial efficiency indicators, Colombian apparel industry, Economic Value Added (EVA), Market Value Added (MVA).

\title{
1. INTRODUCCIÓN
}

El sector de sistema moda, que incluye a la industria de confecciones, hace parte de los 18 sectores priorizados por la más reciente política de desarrollo productivo -Conpes 3866 de 2016-, para ejecutar programas que promuevan su productividad y competitividad dado su tradición, impacto en la producción, exportación, empleo y su potencialidad para generar productos con mayor valor agregado (Colombia productiva, 2018). De ahí que empresarios, entidades del Estado y la academia requieran conocer su desempeño financiero $y$, específicamente, si esta industria es rentable y creadora de valor económico y qué factores han incidido para este comportamiento. Esta inquietud es la que se pretende resolver en este artículo, mediante un análisis financiero para el período 20102016. Se utiliza información contable y de mercado para evaluar su crecimiento, la eficiencia en el uso de los activos, la eficacia en la gestión operativa y financiera, la efectividad para generar utilidad sobre la inversión y, también, si genera EVA.

El contenido del artículo se presenta de la siguiente manera: primero, se plantea el marco teórico, definiendo los indicadores financieros apropiados para evaluar el desempeño financiero de un sector industrial; segundo, la metodología, indicando las características, alcance y fuentes de información para abordar este estudio; tercero, se presentan los principales resultados de otras fuentes referentes en el ámbito nacional e internacional; cuarto, se analizan los indicadores que evalúan el desempeño financiero de esta industria en el septenio; quinto, se comparan y analizan los resultados con los reportados en fuentes referentes; $y$, finalmente, se concluye sobre los principales hallazgos de esta investigación. 


\section{MARCO TEÓRICO}

La información contable es utilizada por las organizaciones para medir y evaluar su comportamiento financiero, siendo precisamente los indicadores financieros uno de los instrumentos de análisis más ampliamente conocidos: entre ellos tenemos los de crecimiento, eficacia y efectividad.

Los indicadores de crecimiento miden el progreso del sector en relación con las ventas, activos y utilidad neta. Los indicadores de eficiencia miden la intensidad con que las empresas utilizan sus activos para generar ventas (Wild, Subramanyam \& Hasley, 2007, p. 432); los cuales incluyen la rotación de los activos totales, fijos, operacionales, de los inventarios y de la cartera (Rivera, 2004, pp. 35-36). Los indicadores de eficacia permiten medir el efecto que tienen los costos y gastos de las empresas sobre su margen de utilidad, como sucede con las erogaciones que influyen sobre los márgenes de utilidad bruta, operacional, operacional después de impuestos y neta (Rivera, 2004, p. 37). Los indicadores de efectividad miden los beneficios que recibe la empresa y sus propietarios por la inversión realizada; la empresa mediante el rendimiento del activo, ROA, y los propietarios por medio del rendimiento del patrimonio, ROE. En el sistema Dupont se establece que el ROA es el resultado de multiplicar la rotación de activos por el margen de utilidad operacional; mientras que con el sistema Dupont ampliado, el ROE es el producto de la rotación de activos, el margen de utilidad neta y el apalancamiento financiero² (Ross, Westerfield \& Jordan, 2014, pp. 62-63; Rivera, 2004, pp. $38,52)$.

Pese a que los indicadores ROA, ROE y la utilidad por acción (UPA) son los indicadores contables más relevantes para evaluar el desempeño de las empresas, no han dejado de recibir críticas, por no considerar el riesgo, ni el costo de capital propio ${ }^{3}$, como, también, por estar expuestos a ser manipulados, entre otros (Salaga, Bartosova \& Kicova, 2015, p. 485), lo que ha llevado a que surjan novedosos modelos gerenciales basados en el valor, que buscan medir el desempeño mediante cálculo de la utilidad residual; el más conocido es el EVA (Worthington \& West, 2001, p. 4).

El EVA es la utilidad residual, resultante de restar de la utilidad operacional después de impuestos un cargo por la utilización del capital (Stewart, 2000, p. 164), tal como se expresa en la ecuación (1):

$$
E V A_{t}=U O D I_{t}-\text { Cargo de capital }
$$

donde $U O D I_{t}$ es la utilidad después de impuestos en el período $t$, y el cargo de capital en el período $t$ es igual a:

$$
\text { Cargo de capital } t=\left(A N O_{t-1}\right)\left(K o_{t}\right)
$$

\footnotetext{
${ }^{2}$ Apalancamiento financiero $=$ activo total bruto promedio $/$ patrimonio promedio.

3 Para Joel M. Stern, uno de los dos socios creadores del EVA, «ese es un problema muy grande porque muchas empresas son criticadas por ser "lucrativas", pero si se incluye un costo de capital propio, las ganancias reportadas disminuyen enormemente para reflejar el uso del capital de los inversores. Lo ideal sería que la cuenta de resultados informara un número que refleje el valor creado por el accionista, o lo que llamamos EVA por "valor económico agregado" o MVA por "valor agregado de mercado", aunque preferiría "valor agregado de gestión" porque se ha confiado a la administración con una cierta cantidad de capital y deben ser responsables de crear valor por encima de esa cantidad de capital» (O`Byrne, 2014, p. 40).
} 
siendo $A N O_{t-1}$ el activo neto operacional al principio del período. Esta variable consiste en la sumatoria del capital de trabajo neto operativo $K T N O_{t-1}$ y del activo fijo neto operacional $A F N O_{t-1}$ :

$$
A N O_{t-1}=K T N O_{t-1}+A F N O_{t-1}
$$

El $K T N O_{t-1}$ es la diferencia entre los activos corrientes y los pasivos corrientes sin costo explícito. El $A F N O_{t-1}$ resulta de restar a los activos fijos operacionales su depreciación.

El $K o_{t}$ es el costo de capital medio ponderado del período $t$; según Modigliani \& Miller (1963, p. 441), se calcula así:

$$
K o_{t}=K_{i}(1-t)+K_{e}(1-L)
$$

Donde $K_{i}$ es el costo de la deuda, pero dado que los intereses son deducibles de la base gravable de la empresa, el costo de la deuda después de impuestos queda expresado como $K_{i}(1-t)$; la $t$ representa la tasa de impuestos de la empresa.

$L$ es el nivel de endeudamiento que resulta de dividir la deuda con costo explícito entre el activo neto operacional $A N O$.

$K_{e}$ es el costo del capital propio o costo de oportunidad, y dada la complejidad para determinarlo en empresas que no cotizan en bolsa, en los siguientes párrafos se indica la forma de calcularlo, siguiendo a Rivera \& Alarcón (2012, pp. 89-90).

Según el modelo de valoración de activos de capital (CAPM), el costo del capital propio, $K_{e}$, es igual a la tasa libre de riesgo, $R_{f}$, más el riesgo sistemático de la empresa o sector $\beta$ multiplicado por la prima de riesgo de mercado $\left(R_{m}-R_{f}\right)$, que resulta de la diferencia entre el rendimiento del mercado, $R_{m}$, y la tasa libre de riesgo. Por lo tanto, $K_{e}$ es equivalente a:

$$
K_{e}=R_{f}+\beta\left(R_{m}-R_{f}\right)
$$

Se asume que el costo de oportunidad de los accionistas de una empresa o sector de un país emergente sería muy similar al que tuviera en un país desarrollado referente, con mercados bursátiles más competitivos y eficientes ${ }^{4}$; más una prima por riesgo país, $R P$, por tener la inversión en un país emergente. Esto evitaría las restricciones teóricas y técnicas para el cálculo de la beta en países emergentes, como Colombia, que mantienen un mercado de renta variable pequeño, ilíquido y concentrado. De acuerdo con lo anterior, el costo de oportunidad de la empresa (o sector) del país emergente sería igual a:

$$
K_{e}=R_{f}+\beta\left(R_{m}-R_{f}\right)+R P
$$

\footnotetext{
${ }^{4}$ Normalmente se toma a Estados Unidos de América como país de referencia.
} 
Pero dado que $\beta$ del modelo CAPM representa el riesgo total de la empresa o sector, $\beta_{L}$, que incluye el riesgo sistemático operativo y financiero; y en lo que se considera que habría afinidad entre la empresa o sector emergente y del país desarrollado es en el riesgo operativo, $\beta_{u}$, entonces se hace necesario calcularlo así:

$$
\beta_{u}=\beta_{L} /\left[1+(1-t)\left(\frac{D}{S}\right)\right]
$$

Los datos requeridos para aplicar la ecuación (7) son tomados de la empresa o sector del país desarrollado referente. El índice $\left(\frac{D}{S}\right)$ es el apalancamiento financiero total, que resulta de dividir la deuda, $D$, sobre el capital propio, $S$.

Posteriormente, se calcula la $\beta_{L}$ de la empresa o sector del país emergente con el objeto de considerar la protección fiscal de su propio apalancamiento, empleando la siguiente fórmula:

$$
\beta_{L}=\beta_{u}\left[1+(1-t)\left(\frac{D}{s}\right)\right]
$$

La información requerida para aplicar la ecuación (8) es tomada de la empresa o sector del país emergente.

Con la beta total, $\beta_{L}$, de la empresa o sector del país emergente, se determina $K_{e}$ utilizando la fórmula (6), y obteniendo la información de $R_{f}, \beta_{L}$ y $R_{m}$ del mercado de valores del país desarrollado.

Pero el $K_{e}$ de la ecuación (6) está expresado en moneda extranjera del país desarrollado, por lo tanto, se debe convertir en moneda local; de la siguiente forma:

$$
K_{\text {emondeda local }}=\left[\left(1+K_{\text {e moneda extranjera }}\right)(1+\text { Devaluación })\right]-1
$$

Si se considera una devaluación en condiciones de paridad cambiaria, se utiliza la siguiente fórmula:

$$
\text { Devaluación }=\left[\frac{1+\text { inflación intena }}{1+\text { inflación externa }}\right]-1
$$

Stewart (2000, p. 163) propone una segunda forma de calcular el EVA, que se presenta a continuación:

$$
E V A=\left(A N O_{t-1}\right)\left[\left(U A I I_{t}\right)(1-t) /\left(A N O_{t-1}\right)-\left(K o_{t}\right)\right]
$$

donde $\left(U A I I_{t}\right)(1-t) / A N O_{t-1}$ es el rendimiento después de impuestos del activo neto operacional. A la diferencia entre el rendimiento después de impuestos del activo neto operacional ANO y el costo de capital medio ponderado $K o_{t}$, se le conoce como el porcentaje de utilidad o pérdida residual. La $U A I I_{t}$ es la utilidad antes de impuestos e intereses.

Al traer a valor presente el EVA de varios años, se obtiene el valor de mercado agregado VMA del período evaluado; que se puede expresar de la siguiente forma: 


$$
\mathrm{VMA}=\sum_{\mathrm{j}=1}^{\mathrm{j}=\mathrm{n}} E V A_{\mathrm{j}} /\left(1+\mathrm{Ko}_{\mathrm{j}}\right)^{\mathrm{j}}
$$

\section{METODOLOGÍA}

Para conocer el desempeño financiero de la industria de confecciones en Colombia en el período 2010-2016, se sigue un enfoque cuantitativo de investigación que permita explicar los factores que influyeron sobre su comportamiento, y se utiliza como método el análisis estático y de tendencias de indicadores contables y de gestión del valor que sean más afines a la evaluación financiera de empresas de un sector manufacturero. Estos resultados son comparados con otros referentes en el ámbito nacional e internacional.

Los indicadores contables seleccionados son los de crecimiento, eficiencia, eficacia y efectividad, en tanto que los indicadores de gestión del valor elegidos son el valor económico agregado, EVA, y el valor de mercado agregado, VMA.

Los indicadores contables y los relacionados con la creación de valor de la industria de confecciones en Colombia se calcularon con base en los estados financieros de las empresas del sector confecciones en Colombia, con CIIU: C1410 (DANE, 2012), de los últimos siete años (2010-2016), de las bases de datos SIREM (Superintendencia de Sociedades, 2017) y Benchmark de BPR Asociados Sales \& Credit Management (2017). De allí se obtuvo información de 184 empresas para 2010, 2013 y 2015; 187 empresas para 2011; 181 empresas para 2012; 196 empresas para 2014; y 180 empresas para 2016. En el anexo se identifica cada una de ellas.

Los comparativos con sectores afines en el medio nacional e internacional se realizan con los indicadores de desempeño financiero que pueden ser obtenidos procesando la información reportada por la Revista Dinero (2011-2017) para el primero y por Damodaran (2017) para el segundo.

\section{RESULTADOS Y DISCUSIÓN}

En la Tabla 1 se presentan algunos indicadores financieros al período 2010-2016 del promedio de las 81 empresas más grandes de Colombia del sector textil, confección, diseño y moda en Colombia.

Estas grandes empresas han crecido en este período, donde solo se ha tenido descensos en las ventas en el 2015 y en los activos y patrimonio entre 2014 y 2015; mientras la utilidad neta declinó al inicio del período, pero a partir de 2014 aumentó (Tabla 1, parte a).

La rotación de activos fluctúa levemente hasta el 2013, y a partir del siguiente año comienza a incrementar y llega a su tope más alto entre 2015 y 2016, con indicador de 1,1 veces, lo que refleja una mejora en la gestión de la eficiencia del uso de los activos en los últimos años (Tabla 1, parte b).

El margen de utilidad neta es positiva en cinco de los siete años estudiados, dejando un promedio en el período de $1,87 \%$; su comportamiento es similar a la utilidad neta, presentando su nivel más alto en el 2016 (3,7 \%) y el más bajo en el 2013 (- 0,6 \%). La eficacia en la gestión de costos y gastos se 
reduce de manera continua hasta la mitad del septenio (2013), posterior a este año se recuperó de manera gradual. Ver Tabla 1, parte c.

Tabla 1. Indicadores contables promedio del sector textil, confección, diseño y moda en Colombia (2010-2016) Table 1. Average financial indicators of the textile, apparel, design, and fashion sector in Colombia (2010-2016)

\begin{tabular}{|c|c|c|c|c|c|c|c|c|}
\hline Indicador & 2010 & 2011 & 2012 & 2013 & 2014 & 2015 & 2016 & Promedio \\
\hline \multicolumn{9}{|c|}{ a. Crecimiento } \\
\hline Ventas (MM\$) & 61.277 & 69.662 & 70.451 & 81.060 & 89.276 & 76.500 & 114.179 & 80.344 \\
\hline Activos (MM\$) & 71.984 & 81.456 & 85.365 & 99.514 & 87.774 & 67.934 & 99.564 & 84.799 \\
\hline Patrimonio (MM\$) & 39.078 & 43.226 & 46.601 & 54.867 & 41.884 & 32.313 & 43.603 & 43.082 \\
\hline \multicolumn{9}{|c|}{ b. Eficiencia } \\
\hline $\begin{array}{l}\text { Rotación de activos } \\
\text { totales }\end{array}$ & 0,9 & 0,9 & 0,8 & 0,8 & 1,0 & 1,1 & 1,1 & 0,9 \\
\hline $\begin{array}{l}\text { Utilidad neta /ventas } \\
\text { (\%) }\end{array}$ & 3,3 & 1,7 & c. Eficaci & $-0,6$ & 2,0 & 3,4 & 3,7 & 1,9 \\
\hline \multicolumn{9}{|c|}{ d. Endeudamiento } \\
\hline $\begin{array}{l}\text { Apalancamiento } \\
\text { financiero (\%) }\end{array}$ & 184,2 & 188,4 & 183,2 & 181,4 & 209,6 & 210,2 & 228,3 & 197,9 \\
\hline \multicolumn{9}{|c|}{ e. Efectividad } \\
\hline ROE (\%) & 5,2 & 2,8 & $-0,7$ & $-0,9$ & 4,3 & 8,1 & 9,7 & 4,1 \\
\hline Número de empresas & 109 & 102 & 99 & 86 & 69 & 51 & 52 & 81 \\
\hline
\end{tabular}

Fuente: elaboración propia, con datos de la Revista Dinero (2011, 2012, 2013, 2014, 2015, 2016 y 2017). Nota: MM\$ denota cifras en millones de pesos colombianos.

El apalancamiento financiero en promedio fue de 197,9\%, se evidencia una tendencia a reducirse en la primera parte de septenio, pero cambia de orientación a partir del 2014 donde crece hasta llegar a su punto más alto en el 2016 con un 228,3 \%, como se observa en la Tabla 1, parte d.

El rendimiento promedio del patrimonio es de 4,1\%, presentando una tendencia muy parecida al margen de utilidad en cada uno de los años, y al del apalancamiento financiero, excepto en 2011, aunque apartándose un poco de la orientación de la rotación de activos, salvo algunos años. Con lo que se puede inferir que el comportamiento de la efectividad depende primordialmente de la eficacia, que es ampliada por el apalancamiento financiero (Tabla 1, parte e).

De la información extraída de Damodaran (2017) de las empresas del sector confecciones en los Estados Unidos de América en el período 2010-2016 , se hallan los indicadores de desempeño financiero presentados en la Tabla 2.

\footnotetext{
5 Damodaran (2017) toma datos de Bloomberg, Morningstar, Capital IQ y Compustat que reúnen información de empresas que cotizan en bolsa.

Se toma como referencia el sector confecciones de Estados Unidos de América porque es un mercado al cual la industria de confecciones de Colombia llega con un porcentaje importante de sus exportaciones, y compite por aumentar su participación en este mercado, lo que hace pertinente saber qué tanta efectividad financiera tiene esta industria para afrontar este reto; según el DANE (2019a), durante el período 2010-2016 en promedio el 33,51 \% de las exportaciones tuvieron como destino a Estados Unidos de América, donde la participación promedio del sector confección de prendas de vestir sobre el total de exportaciones fue del 1,1 $\%$ (DANE, 2019b).
} 
Tabla 2. Indicadores promedio del sector confecciones en Estados Unidos de América

Table 2. Average indicators of the apparel sector in the United States

\begin{tabular}{|c|c|c|c|c|c|c|c|c|}
\hline Indicador & 2010 & 2011 & 2012 & 2013 & 2014 & 2015 & 2016 & Promedio \\
\hline EVA (MMUS\$) & 862 & 1.441 & 1.482 & 4.909 & 4.398 & 3.613 & 3.596 & 2.900 \\
\hline UODI (MMUS\$) & 2.882 & 3.849 & 4.119 & 7.778 & 7.105 & 6.616 & 6.132 & 5.497 \\
\hline Cargo de capital (MMUS\$) & 2.020 & 2.408 & 2.637 & 2.869 & 2.707 & 3.003 & 2.536 & 2.597 \\
\hline ANO (MMUS\$) & 22.578 & 27.445 & 29.710 & 35.091 & 39.209 & 41.387 & 41.409 & 33.833 \\
\hline Ko (\%) & 8,9 & 8,8 & 8,9 & 8,2 & 7,1 & 7,5 & 6,5 & 8,0 \\
\hline UODI/ANO (\%) & 12,8 & 14,0 & 13,9 & 22,2 & 18,1 & 16,0 & 14,8 & 16,0 \\
\hline UAII (MMUS\$) & 3.642 & 4.586 & 5.058 & 8.670 & 7.997 & 7.679 & 6.886 & 6.360 \\
\hline Impuestos (MMUS\$) & 759 & 737 & 939 & 892 & 892 & 1.063 & 754 & 862 \\
\hline Ke $(\%)$ & 9,7 & 9,7 & 9,7 & 8,8 & 7,9 & 8,6 & 7,5 & 8,8 \\
\hline Ki (\%) & 5,3 & 4,4 & 3,8 & 6,0 & 3,7 & 4,0 & 4,0 & 4,5 \\
\hline L (\%) & 13,6 & 15,5 & 12,1 & 17,6 & 17,2 & 21,8 & 25,5 & 17,6 \\
\hline $\mathrm{t}(\%)$ & 20,9 & 16,1 & 18,6 & 10,3 & 11,2 & 13,8 & 11,0 & 14,5 \\
\hline VMA a 1-1-2010 (MMUS\$) & 14.414 & & & & & & & \\
\hline $\mathrm{ROE}$ & 11,8 & 13,7 & 16,0 & 17,9 & 18,5 & 14,5 & 14,4 & 15,2 \\
\hline Número de empresas & 48 & 57 & 54 & 70 & 64 & 63 & 58 & 59 \\
\hline
\end{tabular}

Este sector crea valor económico en todos los años, de manera creciente, hasta el 2013, para luego descender. Este comportamiento va acorde con la orientación de la UODI, y esta a su vez sigue la misma tendencia de la utilidad antes de intereses e impuestos (UAII). El cargo de capital también, sigue la misma trayectoria del EVA, salvo en el 2015, tendencia que resulta de un crecimiento continuo del ANO, y un comportamiento fluctuante del Ko. El indicador Ko oscila todo el período, mientras que UODI/ANO oscila hasta el 2013, luego decrece; no obstante, Ko es inferior a UODI/ANO, lo que explica por qué se crea valor económico en cada año, generando un valor de mercado agregado en el septenio de US\$ $14.414 \mathrm{MM}$.

\subsection{Desempeño financiero de la industria de confecciones en Colombia}

En este apartado se calcula y analiza los indicadores de crecimiento, eficiencia, efectividad y de gestión de valor de la industria de confecciones en Colombia.

\subsubsection{Evaluación del crecimiento}

Se observa un crecimiento continuo de los activos y ventas, con una caída en el 2015, y altibajos en la utilidad neta (Tabla 3).

Tabla 3. Activos, ventas y utilidad neta promedio de la industria

Table 3. Assets, sales, and average net profit of the industry

\begin{tabular}{lrrrrrrrr}
\hline & 2010 & 2011 & 2012 & 2013 & 2014 & 2015 & 2016 & Promedio \\
\hline Activos & 17.976 & 19.706 & 21.558 & 24.945 & 26.115 & 15.852 & 32.561 & 22.673 \\
Ventas & 16.410 & 18.260 & 19.387 & 22.963 & 23.438 & 16.617 & 31.794 & 21.267 \\
Utilidad neta & 331 & 485 & 454 & 661 & 389 & 549 & 802 & 524 \\
\hline Nente: elaboración propia con base en SIREM (Superintendencia de Sociedades, 2017) y Benchmark de BPR Asociados \\
\multicolumn{7}{c}{ Sales \& Credit Management (2017). } \\
\multicolumn{7}{c}{ Nota: MM denota cifras en millones de pesos colombianos. }
\end{tabular}




\subsubsection{Evaluación de la eficiencia}

La rotación de cartera ( 4,0 veces), de inventario (2,5 veces) y la rotación de activos fijos (0,6 veces) presentan comportamientos diferentes: el primero después de oscilar, tiende a aumentar a partir del 2013; el segundo desciende a lo largo del período, con un ligero ascenso en el 2015; mientras el tercero varía su orientación en el septenio. Estos comportamientos contrastan con la rotación de activos totales $(0,9)$ que permanece casi constante (Tabla 4 ). Una alta rotación de activos fijos que supera la cartera y los inventarios insinúa una menor eficiencia en la gestión de los activos corrientes.

Tabla 4. Indicadores de eficiencia

Table 4. Efficiency indicators

\begin{tabular}{lccccccccc}
\hline & 2010 & 2011 & 2012 & 2013 & 2014 & 2015 & 2016 & Promedio \\
\hline & & \multicolumn{7}{c}{ Rotación (veces) } \\
Rotación de cartera & 3,4 & 3,8 & 3,7 & 4,0 & 4,1 & 5,2 & ND & 4,0 \\
Rotación de inventario & 2,8 & 2,6 & 2,6 & 2,4 & 2,3 & 2,4 & 2,3 & 2,5 \\
Rotación de activos fijos & 4,8 & 5,5 & 5,4 & 5,6 & 5,4 & 7,2 & 3,5 & 5,4 \\
Rotación de activos totales & 0,9 & 0,9 & 0,9 & 0,9 & 0,9 & 1,0 & 1,0 & 0,9 \\
\hline
\end{tabular}

Fuente: elaboración propia con base en SIREM (Superintendencia de Sociedades, 2017) y Benchmark de BPR Asociados Sales \& Credit Management (2017).

\subsubsection{Evaluación de la eficacia}

En la Tabla 5 se puede observar que los márgenes promedio de utilidad bruta (32,5\%), de utilidad operacional $(6,1 \%)$ y de utilidad neta $(2,5 \%)$ fluctuaron durante los siete años, con una mayor estabilidad en el primero que presenta un crecimiento entre 2013-2015, seguida del segundo que incrementa entre 2015-2016. Estas variaciones demuestran correlaciones positivas en la mayor parte del septenio. Las diferencias de los márgenes señalan que los costos de ventas $(67,5 \%$ ) y los gastos de administración y ventas (26,4\%), en su orden, son las dos erogaciones que más influyen en la gestión eficaz de este sector.

Tabla 5. Indicadores de eficacia

Table 5. Efficacy indicators

\begin{tabular}{lccccccccc}
\hline & 2010 & 2011 & 2012 & 2013 & 2014 & 2015 & 2016 & Promedio \\
\hline & & & \multicolumn{7}{c}{ Márgenes (\%) } \\
Margen de utilidad bruta & 29,4 & 30,0 & 29,8 & 32,8 & 33,7 & 38,3 & 33,1 & 32,5 \\
Margen de utilidad operacional & 5,9 & 6,8 & 4,8 & 5,9 & 5,7 & 6,8 & 7,1 & 6,1 \\
Margen de utilidad neta & 2,0 & 2,7 & 2,3 & 2,9 & 1,7 & 3,3 & 2,5 & 2,5 \\
\hline
\end{tabular}

Fuente: elaboración propia con base en SIREM (Superintendencia de Sociedades, 2017) y Benchmark de BPR Asociados Sales \& Credit Management (2017).

\subsubsection{Evaluación de la efectividad}

El ROA promedio del septenio fue de 4,5\%, siendo resultado de la combinación entre el margen de UODI $(4,7 \%)$ y la rotación de activos totales ( 0,9 veces). El ROA sigue la misma tendencia del margen de UODI, que es fluctuante durante todo el período, indicando que el comportamiento de la efectividad lo determina la eficacia en la gestión de los costos y gastos (Tabla 6). 
Tabla 6. Indicadores de efectividad

Table 6. Effectiveness indicators

\begin{tabular}{|c|c|c|c|c|c|c|c|c|}
\hline & 2010 & 2011 & 2012 & 2013 & 2014 & 2015 & 2016 & Promedio \\
\hline & \multicolumn{8}{|c|}{ a. ROA: Sistema Du Pont } \\
\hline ROA (\%) & 4,3 & 5,0 & 3,1 & 4,3 & 4,0 & 5,1 & 5,5 & 4,5 \\
\hline $\begin{array}{l}\text { Margen de utilidad operacional después de } \\
\text { impuestos (\%) }\end{array}$ & 4,8 & 5,4 & 3,4 & 4,7 & 4,5 & 4,8 & 5,6 & 4,7 \\
\hline \multirow[t]{2}{*}{ Rotación de activos totales (veces) } & 0,9 & 0,9 & 0,9 & 0,9 & 0,9 & 1,0 & 1,0 & 0,9 \\
\hline & \multicolumn{8}{|c|}{ b. ROE: Sistema Du Pont Ampliado } \\
\hline ROE (\%) & 3,9 & 5,0 & 4,1 & 5,1 & 3,1 & 7,8 & 6,1 & 5,0 \\
\hline Margen de utilidad neta (\%) & 2,0 & 2,7 & 2,3 & 2,9 & 1,7 & 3,3 & 2,5 & 2,5 \\
\hline Rotación de activos totales (veces) & 0,9 & 0,9 & 0,9 & 0,9 & 0,9 & 1,0 & 1,0 & 0,9 \\
\hline Apalancamiento financiero (\%) & 210,0 & 204,6 & 194,6 & 193,4 & 207,6 & 226,7 & 248,7 & 212,2 \\
\hline
\end{tabular}

Fuente: elaboración propia con base en SIREM (Superintendencia de Sociedades, 2017) y Benchmark de BPR Asociados Sales \& Credit Management (2017).

De otro lado, el ROE promedio fue de 5,0 \%, resultado del producto de los siguientes indicadores: el margen de utilidad neta $(2,5 \%)$, la rotación de activos totales (0,9 veces) y el apalancamiento financiero (212,2\%). En el septenio, el ROE presenta altibajos más pronunciados que el ROA (el primero fluctúa entre $7,8 \%$ y 3,1 \%; mientras el segundo, entre $5,5 \%$ y 3,1\%), esto obedece a las variaciones del apalancamiento financiero, ya que el margen de utilidad neta varía menos que la utilidad operacional después de impuestos (la primera oscila entre 1,7 \% y 2,9 \% y la segunda entre $3,4 \%$ y 5,6 \%). El comportamiento del ROE sigue la tendencia del margen de utilidad neta, dado que la rotación de activos se mantiene estable y el apalancamiento financiero conserva una correlación negativa con el ROE en varios años; con lo que se puede afirmar que el cambio de la efectividad de esta industria obedece principalmente al comportamiento de la eficacia en la gestión de costos y gastos.

\subsubsection{Evaluación del valor económico agregado de la industria de confecciones en Colombia: período 2010-2016}

La empresa promedio de la industria de confecciones en Colombia ha destruido valor económico agregado en cada año, aunque de manera oscilante, que es consecuencia del comportamiento fluctuante del cargo de capital y de la UODI (Tabla 7).

El cargo de capital cambia conforme a las fluctuaciones anuales del Ko y del aumento casi permanente del ANO, que solo disminuyó en 2015 (Tabla 7, parte a). El ANO sigue la misma tendencia del KTNO y del AFNO. La superioridad del AFNO sobre el KTNO se explica porque es un sector industrial (Tabla 7, parte c).

La UODI presenta una correlación positiva con la UAll, mientras que los impuestos crecen año tras año. A su vez, la UAll no presenta el mismo comportamiento de los rubros que la conforman: ventas, costo de ventas, utilidad bruta, gastos de administración y ventas que ascendieron gradualmente en el período, salvo una caída en el 2015 (Tabla 3 y Tabla 7, parte b).

Los inductores del Ko indican que su comportamiento depende principalmente de la orientación del Ke con el que mantiene una correlación positiva; y en segundo término con la tasa de impuestos que varía de manera similar hasta el antepenúltimo año. Aunque la oscilación de Ki es contraria a la del 
porcentaje de endeudamiento, no siguen el comportamiento de Ko en muchos años, como se exhibe en la Tabla 7, parte d.

Al evaluar el porcentaje de utilidad o pérdida residual se observa que la relación UODI/ANO fue inferior a Ko en los siete años, lo que explica el por qué esta industria destruye valor en cada año. El indicador UODI/ANO ha variado en todo el septenio, siguiendo la misma tendencia de uno de los dos factores explicativos: el índice UODI/Ventas; el otro factor: Ventas/ANO, permanece casi constante en los primeros cinco años y solo obtiene una variación importante en el 2015 (Tabla 7, parte e).

Tabla 7. EVA promedio por empresa y sus inductores Table 7. Average EVA per company and drivers

\begin{tabular}{|c|c|c|c|c|c|c|c|c|}
\hline & 2010 & 2011 & 2012 & 2013 & 2014 & 2015 & 2016 & Promedio \\
\hline EVA (MM\$) & -791 & -751 & -1.514 & -1.085 & -1.385 & -923 & -1.084 & -1.076 \\
\hline UODI (MM\$) & 781 & 979 & 667 & 1.083 & 1.056 & 804 & 1.781 & 1.022 \\
\hline $\begin{array}{l}\text { Cargo de capital } \\
\text { (MM\$̦) }\end{array}$ & 1.571 & 1.730 & 2.181 & 2.167 & 2.441 & 1.726 & 2.866 & 2.098 \\
\hline \multicolumn{9}{|c|}{ a. Inductores del cargo de capital } \\
\hline ANO (MM\$) & 13.077 & 14.555 & 16.531 & 19.074 & 19.885 & 10.613 & 21.358 & 16.442 \\
\hline Ko (\%) & 12,0 & 11,9 & 13,2 & 11,4 & 12,3 & 16,3 & 13,4 & 12,9 \\
\hline \multicolumn{9}{|c|}{ b. Indicadores del inductor UODI } \\
\hline UAll (MM\$) & 971 & 1.235 & 927 & 1.346 & 1.341 & 1.130 & 2.267 & 1.317 \\
\hline Impuestos (MM\$) & 191 & 255 & 260 & 263 & 286 & 326 & 485 & 295 \\
\hline $\begin{array}{l}\text { Utilidad bruta } \\
\text { (MM\$) }\end{array}$ & 4.832 & 5.486 & 5.776 & 7.539 & 7.909 & 6.373 & 10.533 & 6.921 \\
\hline Costo de venta & & & & & & & & \\
\hline$(\mathrm{MM})$ & 11.578 & 12.774 & 13.611 & 15.423 & 15.529 & 10.245 & 21.260 & 14.346 \\
\hline $\begin{array}{l}\text { Gastos de Admón. } \\
\text { (MM\$) }\end{array}$ & 1.138 & 1.254 & 1.421 & 1.614 & 1.701 & 1.527 & 2.648 & 1.615 \\
\hline $\begin{array}{l}\text { Gastos de venta } \\
\text { (MM\$) }\end{array}$ & 2.723 & 2.998 & 3.428 & 4.579 & 4.867 & 3.716 & 5.859 & 4.024 \\
\hline \multicolumn{9}{|c|}{ c. Indicadores del inductor ANO } \\
\hline KTNO (MM\$) & 5.862 & 6.592 & 7.265 & 8.683 & 9.129 & 5.101 & 9.609 & 7.463 \\
\hline AFNO (MM\$) & 7.215 & 7.963 & 9.266 & 10.391 & 10.756 & 5.512 & 11.749 & 8.979 \\
\hline \multicolumn{9}{|c|}{ d. Indicadores del Inductor Ko } \\
\hline Ke (\%) & 16,3 & 15,3 & 16,7 & 14,6 & 17,3 & 22,5 & 17,4 & 17,2 \\
\hline Ki (\%) & 6,9 & 7,7 & 9,0 & 7,4 & 7,2 & $8,0 \%$ & 11,4 & 8,2 \\
\hline $\begin{array}{l}\text { Deuda con costo } \\
\text { explícito (MMS) }\end{array}$ & 4.680 & 4.863 & 5.252 & 6.649 & 7.624 & 3.793 & 8.280 & 5.877 \\
\hline L (\%) & 35,8 & 33,4 & 31,8 & 34,9 & 38,3 & 35,7 & 38,8 & 35,5 \\
\hline $\mathrm{t}(\%)$ & 36,5 & 34,5 & 36,5 & 28,5 & 42,3 & 37,3 & 37,7 & 36,2 \\
\hline \multicolumn{9}{|c|}{ e. Sistema Dupont del índice UODI/ANO } \\
\hline UODI/ ANO (\%) & 6,0 & 6,7 & 4,0 & 5,7 & 5,3 & 7,6 & 8,3 & 6,2 \\
\hline UODI/Ventas (\%) & 4,8 & 5,4 & 3,4 & 4,7 & 4,5 & 4,8 & 5,6 & 4,7 \\
\hline Ventas/ANO (veces) & 1,3 & 1,3 & 1,2 & 1,2 & 1,2 & 1,6 & 1,5 & 1,3 \\
\hline \multicolumn{9}{|c|}{ f. Valor del mercado agregado } \\
\hline $\begin{array}{l}\text { VMA a 1-1-2010 } \\
\text { (MM\$\$) }\end{array}$ & -4.750 & & & & & & & \\
\hline
\end{tabular}

Fuente: elaboración propia con base en SIREM (Superintendencia de Sociedades, 2017), Benchmark de BPR Asociados Sales \& Credit Management (2017), Superintendencia Financiera (2017) y Damodaran (2017). Nota: MM\$ denota cifras en millones de pesos colombianos. 
El VMA de la industria colombiana de confecciones al 1 de enero de 2010 es de $-\$ 4.750 \mathrm{MM}$, lo que significa que a pesar de que esta industria generó rentabilidades en cada uno de los años, destruyó valor económico durante el septenio (Tabla 7, parte f).

\subsection{Análisis y contrastes de los resultados}

En este aparte se comparan los principales resultados de esta investigación sobre la Industria de Confecciones en Colombia (ICC) con los datos del sector de textil, confección, diseño y moda (SCC) reportados por la Revista Dinero (2011, 2012, 2013, 2014, 2015, 2016 y 2017) ${ }^{6}$ y la información del sector confecciones en los Estados Unidos de América (SCEUA) de la base de datos de Damodaran (2017), en indicadores financieros relacionados con el crecimiento, eficiencia, eficacia, efectividad y el valor económico agregado.

\subsubsection{Crecimiento}

Como se presumía, los valores absolutos promedio de ventas, activos y utilidad neta de las empresas de este estudio, que incluye pymes, son inferiores a los reportados por las grandes empresas en la Revista Dinero (Tablas 1 y 3). Se observa un crecimiento continuo de las ventas, tanto en la ICC como en el SCC, con un bache en el 2015; un aumento permanente de los activos en la ICC y en el SCC, pero con caídas en 2015 en el primero y en el bienio 2014-2015 en el segundo; una variación de la utilidad neta en la ICC, en contraste con las presentadas en el SCC que decae en los primeros cuatro años, pero luego asciende.

\subsubsection{Eficiencia}

La rotación promedio de los activos totales es igual para la ICC y para el SCC con 0,9 veces, y con una mínima mayor estabilidad en la ICC, lo que demuestra que ambos grupos son igual de eficientes en el uso de los recursos, aunque la ICC lo logra con poco de mayor estabilidad (Tablas 1 y 4 ).

\subsubsection{Eficacia}

El promedio del margen de utilidad neta en el septenio fue superior en la ICC, lo que es señal de una menor eficacia en la gestión de control de los costos y gastos por parte de las grandes empresas reportadas por la Revista Dinero, aunque estas últimas logran una mayor estabilidad en su tendencia. En la Tabla 5 se observa que en cada año la ICC presentó márgenes netos positivos, que oscilaban alrededor de 2,5\%, mientras que el SCC arrojó márgenes netos negativos entre 2012-2013, dejando un saldo promedio de $1,9 \%$ en el septenio.

\subsubsection{Apalancamiento financiero}

El apalancamiento financiero promedio de la ICC (212,2 \%) es mayor que el de SCC (197,9\%); ambas con una tendencia a reducirse hasta la mitad del período estudiado, y posteriormente incrementar,

\footnotetext{
${ }^{6}$ Aunque estas empresas cotizan en bolsa, y su tamaño es mayor que el promedio de las empresas colombianas, su dimensión puede influir sobre el desempeño, y sobre el EVA de una organización de manera positiva o negativa, dependiendo especialmente de la eficacia en el uso de los activos.
} 
lo que evidencia la mayor capacidad de la ICC por hacerse a un mayor endeudamiento en casi todos los años analizados, excepto en el 2014; pero, también, los mayores riesgos que asume por tener este índice más alto (Tablas 1 y 6 ).

\subsubsection{Efectividad}

Al cotejar el ROE promedio de la ICC $(5,0 \%)$ del SCC (4,1\%), y del SCEUA $(15,2 \%)$ se aprecia la superioridad del sector confecciones en Estados Unidos de América, que triplica y casi cuadruplica respectivamente el rendimiento del total de empresas de este sector en Colombia y de las grandes empresas listadas por la Revista Dinero. Mientras que la estabilidad en la tendencia de este índice está a favor de la ICC y en contra deI SCC que es la que presenta una mayor variabilidad, además este último es el único que presenta ROE negativos en algunos años (Tablas 1, 2 y 6).

El ROE fue mayor en la ICC que en el SCC, porque dos de los tres factores que lo conforman fueron superiores: el margen neto ( $2,5 \%$ vs $1,9 \%$ ) y el apalancamiento financiero ( $212,2 \%$ vs $197,9 \%$ ), además este último índice siempre tuvo efectos positivos en el ROE de la ICC. (Tablas 1 y 6 ).

\subsubsection{Valor económico agregado}

El EVA de la ICC en Colombia es negativo y oscilante, mientras que en su homólogo estadounidense es positivo y con tendencia creciente (al comienzo) y decreciente (al final) en el septenio, lo que arroja un VMA negativo en Colombia (\$-4.750 MM) y positivo en Estados Unidos de América (\$14.414 MM), como se aprecia en las Tablas 2 y 7.

Al revisar los inductores del EVA se encuentra que el Ko es más alto en todos los años en Colombia, lo cual era de esperarse por el efecto de riesgo país, pero no ocurrió lo mismo con el rendimiento del activo neto operacional, pues contrario a lo esperado, es superado cada año por el SCEUA: el promedio del costo de capital de la ICC es del $12,9 \%$ y en el SCEUA es del $8,0 \%$, en tanto que el promedio del indicador UODI/ANO es de 6,2 \% en la ICC y del 16,0 \% en el SCEUA. Esto explica el por qué en la ICC se obtiene un porcentaje de pérdida residual, mientras que en el SCEUA se llega a un porcentaje de utilidad residual (en promedio fue de $-6,7 \%$ vs $8,0 \%$ ).

\subsubsection{Hallazgos e implicaciones}

Los resultados de este estudio muestran que la industria de confecciones en Colombia ha destruido valor en el período 2010-2016 y esto obedece a que los costos de sus recursos no alcanzan a ser cubiertos por la utilidad operacional después de impuestos que genera; este resultado es contrario a lo mostrado por los indicadores contables ROA y ROE que siempre presentaron resultados positivos. Es posible que por restricciones del mercado y el riesgo país, los costos de los recursos sea el reflejo de un riesgo sistemático, con mayor complejidad de gestionar por parte de los directivos, por eso queda la alternativa de lograr indicadores de efectividad superiores (aumentar el rendimiento del activo neto operacional o el ROE), donde las empresas colombianas de confecciones, también, están en desventaja en relación con el sector homólogo estadounidense, que no solamente lo supera en efectividad, sino en un menor costo de los recursos financieros. 
Para la academia, analistas e investigadores del desempeño financiero sectorial es una prueba más que el análisis de los indicadores contables no siempre guarda coherencia con los indicadores de gestión del valor, y que se hace necesario profundizar el análisis con los indicadores de gestión del valor.

Para los empresarios, inversionistas y demás stakeholders del sector confecciones en Colombia es un referente que les va a permitir comparar la situación de su empresa en particular con el promedio del sector en este período; si es una empresa grande y/o exportadora saber qué tan competitiva es en relación con las de mayor tamaño en Colombia y/o con el sector de confecciones de USA.

El hecho de que en promedio el sector confecciones destruya valor, no solo es desestimulo para propietarios y stakeholders que pone en riesgo su continuidad o al menos su potencial crecimiento, y aunque esto no ocurrió en el septenio analizado, deja una señal que al sector le urge mejorar su efectividad financiera, si quiere mantenerse en un nivel de competitividad que le exige el entorno, de lo contrario corren con el riesgo de desaparecer o ser absorbidas por multinacionales extranjeras.

\section{CONCLUSIONES}

Del análisis realizado a la industria de confecciones en Colombia durante el período 2010-2016, se encontró un crecimiento continuo de sus activos y ventas, con una corrección en el 2015. El comportamiento fluctuante de la efectividad de lograr utilidades sobre el capital a lo largo del septenio obedece principalmente a la eficacia en el control de costos y gastos, dado que la eficiencia en el uso de los activos permaneció casi constante y que el apalancamiento financiero presentó en varios años una correlación negativa con el ROE.

En el septenio resultó ser más efectiva la ICC que el SCC, aunque con una menor estabilidad, lo que muestra un desempeño financiero inferior de las empresas élite de este sector, aunque con un poco menos de riesgo; esto se debió a una mejor eficacia en la gestión del costo y gasto, además de un mayor apalancamiento financiero. Sin embargo, cuando la comparación se hace con su homólogo en EUA se nota una mayor efectividad de este último (ROE de SCEUA: 15,2 \% vs ROE de ICC: 5,0 \% vs. ROE de SCC: $4,1 \%$ ), aunque con una menor estabilidad.

Pese a que en el septenio la ICC obtuvo utilidades contables cada año, y a que su ROA y ROE fueron positivos, destruyó EVA todos los años, dejando como saldo un VMA negativo (\$-4.750 MM). La destrucción fluctuante del EVA en el transcurso del período analizado contrasta con lo sucedido con el sector homólogo en EUA, donde se observa creación del EVA con dos tendencias en el septenio: al principio creciente y después decreciente. El comportamiento del EVA de la ICC en Colombia se ve afectado por las variabilidades de la UODI/ANO y del cargo de capital. El rendimiento del activo operacional siempre fue inferior al costo de capital, lo que explica por qué se destruye valor en cada año. Esto difiere de lo encontrado en el SCEUA, donde todos los años se obtiene un porcentaje de utilidad residual.

Las utilidades contables y el crecimiento que presentó la industria de confecciones en Colombia en el período 2010-2016 no fueron suficientes para lograr el objetivo financiero de creación de valor que define las finanzas corporativas modernas, lo que la pone en desventaja competitiva ante 
sectores homólogos, como el de EUA, haciéndolas propensas a desaparecer o ser absorbidas por multinacionales extranjeras. Con los resultados de estudio se podría inferir que esta industria puede reorientar su rumbo si logra al menos incrementar la rentabilidad de sus activos netos operacionales, ya que la reducción de los costos de los recursos es una tarea más compleja por el riesgo sistemático que genera diversos factores socioeconómicos.

Este artículo presenta los resultados de una investigación del desempeño financiero de la industria de confecciones en Colombia, sin hacer distinción de su edad, tamaño, ubicación geográfica, naturaleza jurídica y otros factores, que en la literatura financiera y medio empresarial son reconocidos como importantes para evaluar poblaciones más homogéneas y corroborar e identificar nuevos comportamientos, variables que influyen sobre su comportamiento financiero, por lo que este trabajo puede considerarse como un inicio para futuros estudios enfocados a estos nuevos grupos poblacionales.

\section{REFERENCIAS}

BPR Asociados Sales \& Credit Management (2017). Benchmark database. Recuperado de http://bck.securities.com/mainview?sector_id=9999028\&sv=BCK\&pc=CO.

Colombia productiva. (2018). ¿Qué hace el PTP? Recuperado de https://www.ptp.com.co/conozcanos/que-hace-ptp

Consejo Nacional de Política Económica y Social. (2016). Política Nacional de Desarrollo Productivo: CONPES $3866 . \quad$ Recuperado de https://colaboracion.dnp.gov.co/CDT/Conpes/Econ\%C3\%B3micos/3866.pdf

Damodaran, A. (2017). Base de datos en línea. Recuperado de http://pages.stern.nyu.edu/ adamodar/

DANE (2012). Clasificación industrial internacional uniforme de todas las actividades económicas. Revisión 4 adaptada para Colombia. CIIU Rev. 4 A.C. Recuperado de https://www.dane.gov.co/files/nomenclaturas/CIIU_Rev4ac.pdf

DANE (2019a). Colombia, destino de las exportaciones 1994-2019p. Recuperado de https://www.dane.gov.co/index.php/estadisticas-por-tema/comercio-

internacional/exportaciones

DANE (2019b). Colombia, exportaciones totales, según CIIU Rev. 4. 1995 - 2019p. Recuperado de https://www.dane.gov.co/index.php/estadisticas-por-tema/comerciointernacional/exportaciones

Modigliani, F. \& Miller, M. (1963). Corporate income taxes and the cost of capital: a correction. The American Economic Review, 53, 433-443.

O`Byrne, S. (2014). A Look Back at the Beginnings of EVA and Value-Based Management: An Interview with Joel M. Stern. Journal of Applied Corporate Finance, 26(1), 39-46. 
Revista Dinero (2011). 5000 mil empresas. 234-235.

Revista Dinero (2012). 5000 mil empresas. 226-228.

Revista Dinero (2013). 5000 mil empresas. 228-230.

Revista Dinero (2014). 5000 mil empresas. 234-236.

Revista Dinero (2015). 5000 mil empresas. 198.

Revista Dinero (2016). 5000 mil empresas. 180-182.

Revista Dinero (2017). Ranking de las 5 mil empresas. 140-142.

Rivera, J. (2004). Introducción a la administración financiera: fundamentos y aplicaciones para crear valor. Recuperado de https://books.google.com.co/books/about/Introducci\%C3\%B3n_a_la_administraci\%C3\%B3n_fi nan.html?id=t0cPjwEACAAJ\&redir_esc=y

Rivera, J. \& Alarcón, D. (2012). El cargo de capital en la evaluación del desempeño financiero de empresas innovadoras de confecciones de Cali. Estudios Gerenciales, 38(123), 85-100.

Ross, S.; Westerfield, R. \& Jordan, B. (2014). Fundamentos de finanzas corporativas. (10a. edición). México: McGraw-Hill Interamericana.

Salaga, J., Bartosova, V. \& Kicova, E. (2015). Economic value added as a measurement tool of financial performance. Procedia Economics and Finance, 26, 484-489.

Stewart, B. (2000). En busca del valor. Barcelona: Ediciones Gestión 2000.

Superintendencia de Sociedades (2017). Base de datos SIREM: estados financieros y gastos de intereses. Recuperado de http://www.supersociedades.gov.co/asuntos-economicos-ycontables/estudios-y-supervision-por-riesgos/SIREM/Paginas/default.aspx

Superintendencia Financiera de Colombia (2017). Tasa de interés y desembolsos por modalidad de crédito. Recuperado de https://www.superfinanciera.gov.co/jsp/loader.jsf?|Servicio=Publicaciones\&|Tipo=publicacione s\&lFuncion=loadContenidoPublicacion\&id=60955

Wild, J.; Subramanyam, K \& Hasley, R. (2007). Análisis de estados financieros. (9a. edición). México: McGraw-Hill Interamericana.

Worthington, A. \& West, T. (2001). Economic value-added: A review of the theoretical and empirical literature. Asian Review of Accounting, 9(1), 67-86. 